\title{
Finite-Temperature Spin Dynamics in a Perturbed Quantum Critical Ising Chain with an $E_{8}$ Symmetry
}

\author{
Jianda Wu, ${ }^{1}$ Márton Kormos, ${ }^{1,2}$ and Qimiao $\mathrm{Si}^{1}$ \\ ${ }^{1}$ Department of Physics and Astronomy, Rice University, Houston, Texas 77005, USA \\ ${ }^{2}$ MTA-BME "Momentum" Statistical Field Theory Research Group, H-1111 Budapest, Budafoki út 8, Hungary
}

(Received 29 August 2014; published 9 December 2014)

\begin{abstract}
A spectrum exhibiting $E_{8}$ symmetry is expected to arise when a small longitudinal field is introduced in the transverse-field Ising chain at its quantum critical point. Evidence for this spectrum has recently come from neutron scattering measurements in cobalt niobate, a quasi-one-dimensional Ising ferromagnet. Unlike its zero-temperature counterpart, the finite-temperature dynamics of the model has not yet been determined. We study the dynamical spin structure factor of the model at low frequencies and nonzero temperatures, using the form factor method. Its frequency dependence is singular, but differs from the diffusion form. The temperature dependence of the nuclear magnetic resonance (NMR) relaxation rate has an activated form, whose prefactor we also determine. We propose NMR experiments as a means to further test the applicability of the $E_{8}$ description for $\mathrm{CoNb}_{2} \mathrm{O}_{6}$.
\end{abstract}

DOI: 10.1103/PhysRevLett.113.247201

PACS numbers: 75.10.Pq, 05.30.Rt, 71.10.Hf

Introduction.-Quantum criticality is a subject of extensive interest in various contexts [1,2]. These range from correlated-electron bulk materials, which can be tuned to the border of magnetism, to systems in low dimensions, where quantum fluctuations are enhanced. The collective fluctuations of a quantum critical point (QCP) often lead to unusual properties. Even in equilibrium, the statics and dynamics are mixed at a QCP. This gives rise to dynamical scaling, while also making it difficult to calculate the fluctuation spectrum. The latter is especially so for the dynamics at nonzero temperatures $(T>0)$ in the "quantum relaxational" regime, which corresponds to small frequencies $\left(\omega \ll k_{B} T / \hbar\right)$ or long times. Indeed, even for the canonical QCP of a transverse-field Ising model in one dimension, it has been challenging to calculate such realfrequency dynamics [3,4].

We are interested here in the one dimensional transverse field Ising model in the presence of a small longitudinal field. The transverse-field-induced QCP in the absence of a longitudinal field [5] has an emergent conformal invariance in the scaling limit [6]. When a small longitudinal field is turned on at the QCP, the excitation spectrum becomes discrete at low energies. The perturbed conformal field theory [7] provided evidence that certain properties of the spectrum of the resulting relativistic field theory and the scattering matrix can be organized in terms of $E_{8}$, an exceptional simple Lie group of rank 8 . The discrete spectrum corresponds to eight particles, whose masses form ratios which are related to the roots of the $E_{8}$ algebra. (For introductory discussions, see Refs. [8,9].) The first two particles describe bound states that are well below the continuum part of the spectrum. Recently, neutron scattering measurements have been carried out in a ferromagnetic cobalt niobate $\mathrm{CoNb}_{2} \mathrm{O}_{6}$, whose $\mathrm{Co}^{2+}$ are coupled in a
quasi-1D way; the experiment identified two excitations whose energy ratios are close to the predicted value, the golden ratio [10].

In this Letter, we study the low-frequency dynamical spin structure factor at finite temperatures using the form factor method [11]. From a theoretical perspective, our calculation provides an illustrative setting to determine the dynamics in the quantum-relaxational regime. For the $E_{8}$ model, the dynamics at finite temperatures have not been systematically studied. From the perspective of the material $\mathrm{CoNb}_{2} \mathrm{O}_{6}$, our study determines the temperature dependence of the NMR relaxation rate. We note that our results bear some similarities with those for another model, the $\mathrm{O}$ (3) nonlinear sigma model [12,13], although our study here benefits from the exactly regularized form factor series $[14,15]$. We also note that a numerical analysis of a generalized transverse-field Ising chain suggests that the $E_{8}$ description survives suitable generalizations of the interactions beyond the nearest-neighbor ferromagnetic coupling [16].

The model.-Consider the Hamiltonian

$$
H_{Z}=-J\left(\sum_{i} \sigma_{i}^{z} \sigma_{i+1}^{z}+g \sum_{i} \sigma_{i}^{x}+h_{z} \sum_{i} \sigma_{i}^{z}\right),
$$

where $\sigma_{i}^{x}$ and $\sigma_{i}^{z}$ are the Pauli matrices associated with the spin components $S^{\mu}=\sigma^{\mu} / 2,(\mu=x, y, z)$, and $i$ marks a site position; in addition, $g$ and $h_{z}$ are the physical transverse and longitudinal fields, respectively, in units of the nearest-neighbor ferromagnetic exchange coupling $J$ between the longitudinal $(z)$ components of the spins. In the absence of the longitudinal field $\left(h_{z}=0\right)$ the system undergoes a quantum phase transition when the transverse field is tuned across its critical value $g=g_{c}=1$ [5]. As is well known, the QCP is described by a $(1+1)$-dimensional 
conformal field theory (CFT) with a central charge 1/2 [6]. More surprising is what happens when a small longitudinal field $h_{z}$ is introduced at the QCP $g=g_{c}$. The action in the continuum limit is given by

$$
\mathcal{A}_{E_{8}}=\mathcal{A}_{c=1 / 2}+h \int d x d \tau \sigma(\tau, x) .
$$

This is an integrable field theory, and is referred to as the $E_{8}$ model because of the aforementioned connection between its properties and the $E_{8}$ group [7,11]. In the above equation, $\tau$ is the imaginary time, $\mathcal{A}_{c=1 / 2}$ stands for the action of the two dimensional CFT with central charge $1 / 2$, and $\sigma(x)$ is a primary field with scaling dimension $1 / 8$. In addition, $h=c J h_{z} / a$, where $a$ is the lattice constant and $c \approx 0.783$ converts between the $\sigma$ field of the continuum theory and its lattice counterpart $\sigma^{z}$ [17]. This describes a scattering theory of eight massive particles, which we will denote by $a, b, c, d, e, f, g, h$ from the lightest to the heaviest. The mass of the lightest particle $\Delta_{a}$ scales with the longitudinal field as $\Delta_{a} \approx 4.405|h|^{8 / 15}$ [18]. The mass of the second lightest particle $\Delta_{b}$ is $\Delta_{a}$ multiplied by the golden ratio $(\sqrt{5}+1) / 2$. These two particles are clearly separated from the two-particle continuum, which appears at energies above $2 \Delta_{a}$.

Local dynamics and NMR relaxation rate.-We focus on the local dynamical structure factor (DSF) of the $E_{8}$ model in the low frequency and low temperature limit: $\omega \ll \Delta_{a}$ and $T \ll \Delta_{a}$ (hereafter, we set $\hbar=1$ and $k_{B}=1$ ).

A useful means to probe the local DSF is via NMR. The NMR relaxation rate is given by [19]

$$
\frac{1}{T_{1}^{\alpha}}=\frac{1}{2 N} A^{2} \sum_{\beta}^{\prime} S_{\beta \beta}\left(\omega_{0}\right) .
$$

Here, $\alpha$ and $\beta$ label the principal axes, and the primed summation is over the principal axes perpendicular to the field orientation $\alpha ; T_{1}^{\alpha}$ is the spin-lattice relaxation time, $N$ is the number of ions per unit cell, and $\omega_{0}$ is the nuclear resonance frequency. In addition, $A$ describes the hyperfine coupling between the spins of a nucleus and the electrons; while this coupling depends on the wave vector $\mathbf{q}$, the dependence is generically smooth and we will take it as a constant. We will consider the static field of the NMR setup to be the transverse field, $\alpha=x$. Correspondingly, the local DSF of interest to NMR is given by $S_{z z}\left(\omega_{0}\right)+S_{y y}\left(\omega_{0}\right)$. As shown in the Supplemental Material [20], for the model we consider,

$$
S_{y y}(\omega)=\omega^{2} S_{z z}(\omega) /\left(4 J^{2}\right) .
$$

Thus, in the low-frequency regime of interest here, $S_{y y}(\omega)$ is negligible compared with $S_{z z}(\omega)$. In the following, we will therefore only consider $S_{z z}$.

We now turn to the calculation of $S_{z z}(\omega)$ through a systematic form factor expansion. Because the excitation spectrum has a gap, we expect that the leading contributions in the low temperature and low frequency limit come from those associated with the few particle states of the light particles. Indeed, we show below that the dominant contribution comes from the two one-particle states of the lightest particle, which we calculate analytically. The conclusion is confirmed by a numerical calculation for contributions that extend to higher orders.

The form factor series. - Integrable field theory techniques made possible the analytic calculation of matrix elements of local observables in the asymptotic scattering state basis, called form factors. The asymptotic states are eigenstates of the energy and momentum operators. It is convenient to use the standard reparametrization in relativistic theories of a particle's energy and momentum through the rapidity of the particle. In terms of the rapidities $\left\{\theta_{i}\right\}$ of the particles, the energy and momentum eigenvalues of the eigenstate $\left|\theta_{1}^{\alpha_{1}}, \ldots, \theta_{n}^{\alpha_{n}}\right\rangle$ (with $\left\{\alpha_{1}, \ldots, \alpha_{n}\right\}$ marking different types of particles) are

$$
\begin{gathered}
E_{n}=\sum_{i=1}^{n} \Delta_{\alpha_{i}} \cosh \left(\theta_{i}\right), \\
P_{n}=\sum_{i=1}^{n} \Delta_{\alpha_{i}} \sinh \left(\theta_{i}\right) .
\end{gathered}
$$

We denote by $F_{n}^{\sigma}\left(\theta_{1}^{\alpha_{1}}, \ldots, \theta_{n}^{\alpha_{n}}\right)$ the form factors of the primary field $\sigma(t, x)$ in the $E_{8}$ model [cf. Eq. (2)] between the vacuum and an $n$-particle asymptotic state,

$$
F_{n}^{\sigma}\left(\theta_{1}^{\alpha_{1}}, \ldots, \theta_{n}^{\alpha_{n}}\right)=\left\langle 0|\sigma(0,0)| \theta_{1}^{\alpha_{1}}, \ldots, \theta_{n}^{\alpha_{n}}\right\rangle .
$$

The few-particle form factors are explicitly known $[17,24,25]$ and have been used to calculate the static spin-spin correlations of the $E_{8}$ model in the ground state $[17,24]$. Here we study the finite-temperature dynamics by a low-temperature expansion series for integrable field theory $[14,26]$, using a finite-volume regularization [14].

The finite temperature two-point correlation function is given by

$$
C(t, x)=\operatorname{Tr}\left[\frac{e^{-H / T}}{\mathcal{Z}} \mathcal{O}(t, x) \mathcal{O}^{\dagger}(0,0)\right],
$$

where $\mathcal{Z}=\operatorname{Tr} e^{-H / T}$ is the partition function, and we are interested in the local observable operator $\mathcal{O}(t, x)=$ $\sigma(t, x)$. The corresponding DSF is

$$
S(\omega, q)=\int_{-\infty}^{\infty} d x \int_{-\infty}^{\infty} d t C(t, x) e^{i \omega t-i q x},
$$

We insert the complete set of asymptotic states between the operators, yielding a double sum, $C(t, x)=$ $\mathcal{Z}^{-1} \sum_{r, s} C_{r, s}(t, x)$, where 


$$
\begin{aligned}
C_{r, s}(t, x) & =\sum_{\left\{\alpha_{j}\right\},\left\{\alpha_{k}^{\prime}\right\}} \int \frac{d \theta_{1} \ldots d \theta_{r}}{(2 \pi)^{r} r !} \int \frac{d \theta_{1}^{\prime} \ldots d \theta_{s}^{\prime}}{(2 \pi)^{s} s !} e^{-\beta E_{r}} \\
& \times e^{-i t\left(E_{s}-E_{r}\right)} e^{-i\left(P_{r}-P_{s}\right) x}\left|\left\langle\theta_{1}^{\alpha_{1}} \ldots \theta_{r}^{\alpha_{r}}|\mathcal{O}| \theta_{1}^{\prime \alpha_{1}^{\prime}} \ldots \theta_{s}^{\prime \alpha_{s}^{\prime}}\right\rangle\right|^{2} .
\end{aligned}
$$

We use the same set of states to write the partition function as $\mathcal{Z}=\sum_{n=0}^{\infty} \mathcal{Z}_{n}$, where

$$
\mathcal{Z}_{n}=\sum_{\left\{\alpha_{j}\right\}} \int \frac{d \theta_{1} \ldots d \theta_{n}}{(2 \pi)^{n} n !} e^{-\beta E_{n}}\left\langle\theta_{1}^{\alpha_{1}} \ldots \theta_{n}^{\alpha_{n}} \mid \theta_{1}^{\alpha_{1}} \ldots \theta_{n}^{\alpha_{n}}\right\rangle .
$$

In infinite volume all the $\mathcal{Z}_{n}$ 's contain singularities associated with the scalar product of two momentum eigenstates with identical rapidities. Similarly, for the observables we are calculating, $C_{r, s}$ also diverge due to the kinematical poles of the form factors whenever two rapidities in the two sets coincide, $\theta_{i}=\theta_{j}^{\prime}$ [26]. However, the double sums can be reorganized such that the aforementioned singularities cancel each other [14],

$$
C(t, x)=\sum_{r, s=0}^{\infty} D_{r, s}(t, x)
$$

where

$$
\begin{aligned}
& D_{0, s}=C_{0, s}, \\
& D_{1, s}=C_{1, s}-\mathcal{Z}_{1} C_{0, s-1}, \\
& D_{2, s}=C_{2, s}-\mathcal{Z}_{1} C_{1, s-1}+\left(\mathcal{Z}_{1}^{2}-\mathcal{Z}_{2}\right) C_{0, s-2}, \\
& \text {..etc. }
\end{aligned}
$$

The natural small parameter in the series (12) is $e^{-\Delta_{a} / T}$. At low frequencies, the energy conserving Dirac deltas in the Fourier transform Eq. (9) force the two states appearing in the form factors to have nearly equal energy, $E_{r}=\omega+E_{s}$. The magnitude of the Boltzmann factor is then set by the sum of the masses in the "heavier" state, i.e.,

$$
D_{r, s} \sim \exp \left\{-\frac{1}{T} \max \left[\sum_{i=1}^{r} \Delta_{i}, \sum_{i=1}^{s} \Delta_{i}\right]\right\}
$$

Thus, in the regime of interest $\left(T / \Delta_{a} \ll 1\right.$ and $\left.\omega / \Delta_{a} \ll 1\right)$, the expansion series in Eq. (12) is a good perturbation series. In this regime, we can safely truncate the series beyond the terms up to the order of $e^{-2 \Delta_{a} / T}$. Simple counting implies that we only need $D_{0,1}, D_{1,0}, D_{0,2}$, $D_{2,0}, D_{1,1}, D_{1,2}+D_{2,1}, D_{2,2}$ with lightest particles, which we now determine. We also note that the series for the twopoint correlator per se contains a $\delta(\omega)$ piece, which is, however, absent in the connected correlation function of interest here [20].

Leading contributions. $-D_{0,1}$ is the channel between the vacuum and one-particle asymptotic "in" state, and is equal to $C_{0,1}$ from Eq. (13). The corresponding contribution to DSF is

$S_{0,1}(\omega, q)=2 \pi\left|F_{1}^{\sigma}\right|^{2} \int d \theta \delta\left(q-\Delta_{1} \sinh \theta\right) \delta\left(\omega-\Delta_{1} \cosh \theta\right)$,

where $\Delta_{1}$ is the mass of a single particle state, and the oneparticle form factor $F_{1}^{\sigma}(\theta)$ is rapidity independent [17]. Since $\cosh \theta \geq 1$ always holds, for the parameter regime $\omega<\Delta_{a}$ the terms $S_{0,1}$ and $S_{1,0}$ do not contribute. Similarly, the $D_{0, s}$ and $D_{r, 0}$ terms for general $r$ and $s$ also vanish.

The first nontrivial contribution is given by connected parts in $D_{1,1}$, i.e., the term coming from the one-particleone-particle form factors, for which we obtain [20]

$$
S_{1,1}(\omega, q)=\frac{\left|F_{2}^{\sigma}(\alpha+i \pi, 0)\right|^{2}\left(e^{-\beta \Delta_{1} \cosh \theta_{+}}+e^{-\beta \Delta_{1} \cosh \theta_{-}}\right)}{\Delta_{1} \Delta_{2}|\sinh \alpha|},
$$

where $\Delta_{1}$ and $\Delta_{2}$ are the masses of the one-particle states, $\alpha=\operatorname{arccosh}\left[\left(\Delta_{1}^{2}+\Delta_{2}^{2}-\left(\omega^{2}-q^{2}\right)\right) /\left(2 \Delta_{1} \Delta_{2}\right)\right]$ and $\cosh \theta_{ \pm}=\left[\omega\left(\Delta_{1}^{2}-\Delta_{2}^{2}+\omega^{2}-q^{2}\right) \pm 2 q \Delta_{1} \Delta_{2} \sinh \alpha\right] /$ $\left[2 \Delta_{1}\left(q^{2}-\omega^{2}\right)\right]$; hereafter, the symbols that denote the types of particles in the form factor are dropped for notational convenience [Eq. (7)].

The corresponding local DSF is $S_{1,1}(\omega)=$ $\int_{-\infty}^{\infty} S_{1,1}(q, \omega) d q$. Equation (17) implies that, up to $e^{-2 \Delta_{a} / T}$, we need only to consider the channels $a-a$, $b-b$, and $c-c$, as well as $a-b, a-c, b-c$. When $\Delta_{1}=\Delta_{2}=\Delta_{i}(i=a, \ldots, h)$,

$$
\left.S_{1,1}(\omega)\right|_{\Delta_{1}=\Delta_{2}=\Delta_{i}}=\int_{\omega}^{\infty} f(q, \omega) e^{-\left(\Delta_{i} / T\right) g(q, \omega)} d q,
$$

with $f(q, \omega)=\left(2 / \Delta_{i}^{2}\right)\left|F_{2}^{\sigma}(\alpha+i \pi, 0)\right|^{2} /|\sinh \alpha| \quad$ and $g(q, \omega)=-\left(\omega / 2 \Delta_{i}\right)+\left(q / 2 \Delta_{i}\right) \sqrt{1+\left(4 \Delta_{i}^{2} / q^{2}-\omega^{2}\right)}$. We can expand the result for small $\omega$. With the details given in the Supplemental Material [20], we find the result to leading order:

$$
\left.S_{1,1}(\omega)\right|_{\Delta_{1}=\Delta_{2}=\Delta_{a}} \approx\left\{\begin{array}{l}
\frac{2\left|F_{2}^{\sigma}(i \pi, 0)\right|^{2}}{\Delta_{a}} e^{-\Delta_{a} / T}\left\{\ln \frac{4 T}{\omega}-\gamma_{E}+\cdots\right\}\left(\omega \ll T \ll \Delta_{a}\right) \\
\frac{2\left|F_{2}^{\sigma}(i \pi, 0)\right|^{2}}{\Delta_{a}} e^{-\Delta_{a} / T}\left\{\sqrt{\frac{\pi T}{\omega}}-\frac{\sqrt{\pi}}{4}\left(\frac{T}{\omega}\right)^{3 / 2}+\cdots\right\}\left(T \ll \omega \ll \Delta_{a}\right)
\end{array}\right.
$$


where $\gamma_{E}$ is the Euler constant. (The same form applies to the contributions by the other particles $b, \ldots, h$, which are suppressed by their thermal factors.) In deriving this expression, we have replaced $\alpha(\omega, q)$ by $\alpha(\omega=0, q=0)$. This is because the dominant contribution comes from the minimum of the energy dispersion at small momentum; it is well supported by the numerical calculation carried out without this replacement (see below).

We observe that the finite- $T$ local DSF diverges logarithmically as $\omega \rightarrow 0$. This divergence differs from the diffusion form [27] of the inverse square root; this is reasonable given that the total $S_{z}$ is not conserved here. When $\Delta_{1} \neq \Delta_{2}$, the denominator on the right-hand side of Eq. (19) does not have any singularity so there will be no divergence.

Next, we consider $D_{1,2}+D_{2,1}$, the terms with a oneparticle and a two-particle state. Up to the order $\mathcal{O}\left(e^{-2 \Delta_{a} / T}\right)$, we focus on the case when all three particles are the lightest $a$ particle (the other channels $a a-b$ and $a a-c$ are expected to behave similarly), which we find to be [20],

$$
\begin{aligned}
S_{(1,2)+(2,1)}(\omega, q)= & \frac{1}{\pi} \int_{-\infty}^{\infty} \frac{d \theta e^{-\beta \Delta_{a} \cosh \theta}}{\left|\sqrt{(f \tilde{\omega}, \tilde{q}, \theta)-1)^{2}-1}\right|} \\
& \times F_{3}^{\sigma}\left(\theta+i \pi, \ln x_{+}, \ln x_{-}\right) \\
& \times F_{3}^{\sigma}\left(\theta+i \pi, \ln x_{-}, \ln x_{+}\right),
\end{aligned}
$$

where

$$
\begin{aligned}
x_{ \pm}= & \frac{1}{2}(\tilde{\omega}+\cosh \theta+\tilde{q}+\sinh \theta) \\
& \times(1 \pm 2 \sqrt{1-2 / f(\tilde{\omega}, \tilde{q}, \theta)}),
\end{aligned}
$$

and $f(\tilde{\omega}, \tilde{q}, \theta)=\left[(\tilde{\omega}+\cosh \theta)^{2}-(\tilde{q}+\sinh \theta)^{2}\right] / 2$ with $\tilde{\omega}=\omega / \Delta_{a}$ and $\tilde{q}=q / \Delta_{a}$. Our analysis [20] shows no contributions from the range $\tilde{\omega}>\tilde{q} \geq 0$, where $\cosh \theta \sim$ $1 / \tilde{\omega} \gg 1$. In the range $\tilde{\omega} \leq \tilde{q}$, we have $\cosh \theta \gtrsim 2-\tilde{\omega}$, indicating there exists a small region of $\tilde{q}$ where $\cosh \theta$ is slightly smaller than 2 . This contribution is expected to be small, and we confirm this by including the channels $D_{1,2}+D_{2,1}$ in our numerical calculation shown below.

For connected parts in $D_{2,2}$, a similar Jacobian will appear as in the calculation of the equal mass case of Eq. (20), and we will encounter the same logarithmic divergence in the frequency dependence. We find no singular terms beyond the logarithmic divergence [20]. This contribution is therefore suppressed by the thermal weight $e^{-2 \Delta_{a} / T}$. Low-frequency divergences are also expected to come from the $D_{n n}$ terms (at $n>2$ ) with particles of the same mass in the two asymptotic states of the form factors. The fact that $D_{22}$ with the same particle does not contain singularities stronger than $\ln \omega$ is a strong indication that none of the higher terms in the series will give a stronger (e.g., power-law) singularity. We conjecture that the $D_{n n}$ terms at $n>2$ have a similar logarithmic

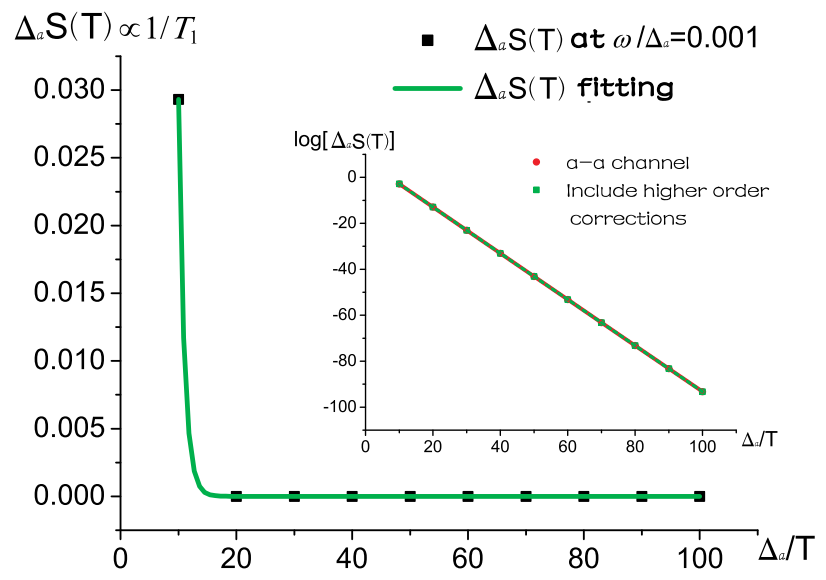

FIG. 1 (color online). The NMR relaxation rate as a function of temperature. The frequency is chosen to be $\omega / \Delta_{a}=0.001$. The temperature dependence is well described by $\Delta_{a} S(T)=$ $631 e^{-\Delta_{a} / T}$. The inset picture shows that channels other than $a-a$ give negligible contributions.

singularity in the frequency dependence, and they are then also negligible compared to $D_{11}$ due to the stronger thermal suppression factor.

Numerical analysis.-Figure 1 shows the results and fit for the NMR relaxation rate as a function of temperature in the range $\Delta_{a} / T \in[10,100]$ at a fixed low frequency $\omega / \Delta_{a}=0.001$ appropriate for the NMR experiments (satisfying $\omega \ll T$ ). The fitting function $\Delta_{a} S(T)=$ $631 e^{-\Delta_{a} / T}$ indicates that the behavior of relaxation rate at low frequency and low temperature region is dominated by the contribution from the $a-a$ channel, as clearly shown in the inset to Fig. 1. The prefactor 631 compares well with the analytical expression associated with $S_{1,1}$ of the lightest $a$ particle, since $2\left|F_{2}^{\sigma}(i \pi, 0)\right|_{\Delta_{1}=\Delta_{2}=\Delta_{a}}^{2} \approx 130$.

We also study the frequency dependence of the local $\mathrm{DSF}$ at fixed temperatures for $T, \omega \ll \Delta_{a}$. Figure 2 shows

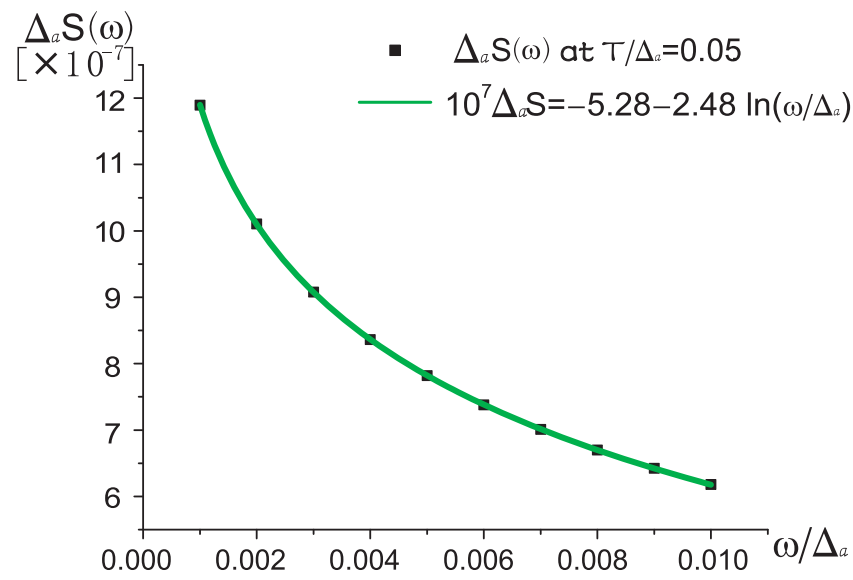

FIG. 2 (color online). The local dynamical structure factor as a function of frequency at a fixed temperature $T / \Delta_{a}=0.05$. The $\omega$ dependence is well described by $10^{7} \Delta_{a} S(\omega)=$ $-5.28+2.48 \ln \left(\Delta_{a} / \omega\right)$. 
the result at a fixed $T / \Delta_{a}=0.05$ with $\omega / \Delta_{a}$ ranging from 0.001 to 0.01 (satisfying $\omega \ll T$. It is well fitted as $10^{7} \Delta_{a} S(\omega)=-5.28-2.48 \ln \left(\omega / \Delta_{a}\right)$, which is in accordance with the asymptotic form Eq. (21).

Discussion.-We conclude that the temperature dependence of the NMR relaxation rate is given by

$$
\frac{1}{T_{1}} \approx \frac{c^{2} b}{\Delta_{a}} \frac{A^{2}}{2 N} e^{-\Delta_{a} / T} ; \quad \Delta_{a} \approx 4.405|h|^{8 / 15} .
$$

In the prefactor, $c \approx 0.783$ is the aforementioned conversion factor between the $\sigma$ field and the lattice spin [17], and $b \approx \ln \left(4 T / \omega_{0}\right)-\gamma_{E}$.

We next consider the implications of our results for $\mathrm{CoNb}_{2} \mathrm{O}_{6}$. The neutron scattering experiments provided evidence for the two lightest particles of the $E_{8}$ spectrum [10]. This has been understood by considering the effect of the interchain coupling in the three-dimensionally ordered state as inducing a longitudinal field [10,28]. Further test of the $E_{8}$ description would be provided by measuring the spin dynamics at finite temperatures. Our study here provides a concrete prediction of the temperature dependence of the NMR relaxation rate in the $E_{8}$ model, which can be used for the desired further test. During the final stage of writing the present manuscript, NMR measurements in $\mathrm{CoNb}_{2} \mathrm{O}_{6}$ have been reported in the higher-temperature quantum critical regime [29]; such measurements at the lower-temperature $E_{8}$ regime should therefore be feasible.

To summarize, we have determined the local dynamical spin structure factor of the perturbed quantum-critical Ising chain at temperatures and frequencies that are small compared to the mass of the lightest $E_{8}$ particle. The frequency dependence shows a logarithmic singularity. Our calculation yields a concrete prediction for the temperature dependence of the NMR relaxation rate, which we have suggested as a means to further test the $E_{8}$ description of the spin dynamics in $\mathrm{CoNb}_{2} \mathrm{O}_{6}$.

We thank R. Coldea, F. H. L. Essler, Gábor Takács and J.H.H. Perk for useful discussions. J.W. and Q.S. acknowledge the support provided in part by the NSF Grant No. DMR-1309531 and the Robert A. Welch Foundation Grant No. C-1411, and M. K. acknowledges that by the Marie Curie IIF Grant PIIF-GA-2012-330076. Q. S. also acknowledges the support of the Alexander von Humboldt Foundation, and the hospitality of the Karlsruhe Institute of Technology and the Institute of Physics of Chinese Academy of Sciences.
[1] Special issue on Quantum Phase Transitions, J. Low Temp. Phys. 161, 1 (2010).

[2] S. Sachdev, Quantum Phase Transitions (Cambridge University Press, Cambridge, England, 2011).

[3] P. Deift and X. Zhou, NATO Adv. Sci. Inst. Ser., Ser. B 320, 183 (1994).

[4] S. Sachdev and A. P. Young, Phys. Rev. Lett. 78, 2220 (1997).

[5] P. Pfeuty, Ann. Phys. (N.Y.) 57, 79 (1970).

[6] A. A. Belavin, A. M. Polyakov, and A. B. Zamolodchikov, Nucl. Phys. B241, 333 (1984).

[7] A. B. Zamolodchikov, Int. J. Mod. Phys. A 04, 4235 (1989).

[8] P. Dorey, Lect. Notes Phys. 498, 85 (1997).

[9] D. Borthwick and S. Garibaldi, Not. Amer. Math. Soc. 58, 1055 (2011).

[10] R. Coldea, D. A. Tennant, E. M. Wheeler, E. Wawrzynska, D. Prabhakaran, M. Telling, K. Habicht, P. Smeibidl, K. Kiefer, Science 327, 177 (2010).

[11] G. Mussardo, Statistical Field Theory (Oxford University Press, New York, 2010).

[12] J. Sagi and I. Affleck, Phys. Rev. B 53, 9188 (1996).

[13] R. M. Konik, Phys. Rev. B 68, 104435 (2003).

[14] B. Pozsgay and G. Takacs, J. Stat. Mech. (2010) P11012.

[15] L. M. Szécsényi and G. Takács, J. Stat. Mech. (2012) P12002.

[16] J. A. Kjäll, F. Pollmann, and J. E. Moore, Phys. Rev. B 83, 020407(R) (2011).

[17] G. Delfino and G. Mussardo, Nucl. Phys. B455, 724 (1995).

[18] Al. B. Zamolodchikov, Int. J. Mod. Phys. A 10, 1125 (1995).

[19] T. Moriya, Prog. Theor. Phys. 28, 371 (1962).

[20] See Supplemental Material at http://link.aps.org/ supplemental/10.1103/PhysRevLett.113.247201, which includes Refs. [21-23], for details of the form factors used in the main text, details of the derivation for several equations of the main text and a discussion of the disconnected contributions to a correlation function presented in the main text.

[21] http://www.sissa.it/ delfino/isingff.html.

[22] Z. Wang and D. Guo, Introduction to Special Functions (Peking University Press, Beijing, China, 2000).

[23] B. Pozsgay and G. Takács, Nucl. Phys. B788, 209 (2008).

[24] G. Delfino and P. Simonetti, Phys. Lett. B 383, 450 (1996).

[25] G. Delfino, P. Grinza, and G. Mussardo, Nucl. Phys. B737, 291 (2006).

[26] F. H. L. Essler and R. M. Konik, J. Stat. Mech. 09 (2009) P09018.

[27] G. F. Giuliani and G. Vignale, Quantum Theory of the Electron Liquid (Cambridge University Press, Cambridge, England, 2005).

[28] S. B. Lee, R. K. Kaul, and L. Balents, Nat. Phys. 6, 702 (2010).

[29] A. W. Kinross, M. Fu, T. J. Munsie, H. A. Dabkowska, G. M. Luke, S. Sachdev, and T. Imai, Phys. Rev. X 4, 031008 (2014). 